\title{
Fürsorge, Gemeinsamkeiten, Pläne - Technik für Fernbeziehungen gestalten
}

\author{
Wei-Chi Chien, Marc Hassenzahl, Eva Lenz
}

Erlebnis und Interaktion, Folkwang Universität der Künste

\section{Zusammenfassung}

Technikvermittelte Kommunikation kann heute so mit emotionalen Aspekten angereichert werden, dass besonders auch für Paare in Fernbeziehungen ein Gefühl von Nähe und Verbundenheit entsteht. Das Verstärken der Gewahrwerdung des Anderen im Alltag, das Schaffen von Möglichkeiten eine "Umarmung" auf die Ferne zu erleben oder das Betonen des emotionalen Ausdrucks sind typische Beispiele dafür. Allerdings scheint es für Paare in Fernbeziehungen oft mehr um das Etablieren neuer beziehungsförderlicher Praktiken zu gehen, als um die Frage, ob man bestehende Kommunikation als emotional empfindet. Auf der Basis psychologischer Literatur und im Rahmen eines autobiographischen Gestaltungsansatzes haben wir drei neue Ansätze für die Gestaltung von Technik zur Unterstützung von Fernbeziehung identifiziert - Fürsorge ermöglichen, Gemeinsamkeiten schaffen, Treffen planen -, erste Konzepte umgesetzt und kritisch erprobt.

\section{$1 \quad$ Einleitung}

Moderne Technik erlaubt es, einfach und kostengünstig mit räumlich weit entfernten Menschen zu kommunizieren. Am 12. März 2015, um 8:58 Uhr zählte die Statistik von http://www.internetlivestats.com 54.663.760 Skype-Anrufe seit Datumswechsel um Mitternacht und bereits 85.092.180 um 13:59 Uhr. Es überrascht nicht, dass in der MenschTechnik-Interaktion Zwischenmenschliches schon lange ein wichtiges Thema ist. Man diskutiert Möglichkeiten der persönlichen Präsenz trotz Entfernung ("Telepresence", Lee 2004) oder versucht, die nonverbale Ebene von Kommunikation in technisch vermittelbare "Signale" zu übersetzen (z.B. Gallace \& Spence 2010).

Ein Review von mehr als 140 Konzepten zur Vermittlung von Verbundenheitsgefühlen (Hassenzahl et al. 2012, siehe auch http://uxrelatedness.blogspot.de/) zeigt sechs Strategien auf, die von Gestaltern angewendet werden: Gewahrwerdung (z.B. den Puls des Partners zu fühlen), emotionaler Ausdruck (z.B. Geheimsprachen), Körperlichkeit (z.B. Umarmungen über die Ferne), Geschenke (z.B. kleine Botschaften), gemeinsame Aktivitäten (z.B. Kochen) und gemeinsame Erinnerungen (z.B. das Blättern in Fotoalben). Allerdings zielen die meis- 
ten Konzepte darauf ab, einzelne Aspekte typischer nonverbaler Kommunikation zu vermitteln, wie z.B. die Körperlichkeit oder die Gewahrwerdung. Dazu kommt, dass sich die Konzepte eher durch die Art der verwendeten Technik unterscheiden, als durch ihren konzeptionellen Ansatz. So ist der zugrunde liegende Mechanismus vom Cubble (Kowalski et al. 2013) beispielsweise kaum anders als das 17 Jahre ältere Konzept Shaker (Strong et al. 1996) - nur in der Verpackung einer neueren Technologie (Abbildung 1).

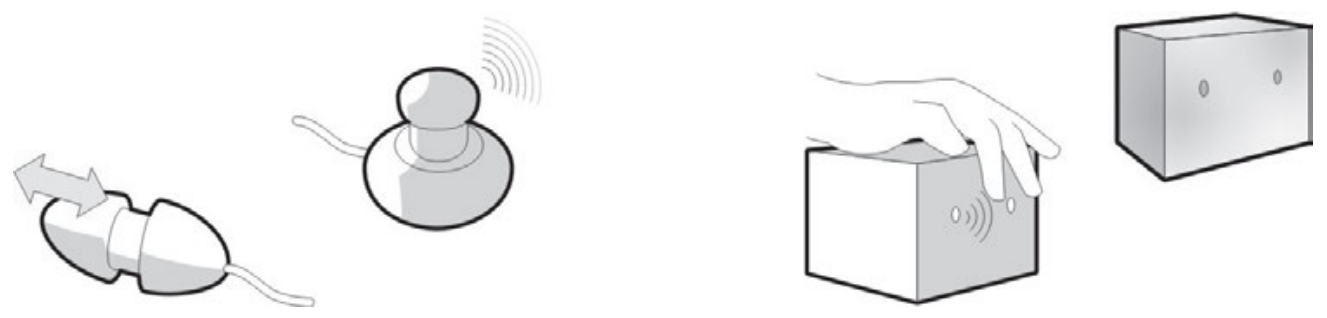

Abbildung 1: Shaker (links) übersetzt eine Bewegung in Vibration. Cubble (recht) übersetzt unterschiedliche Handbewegungen in sich verändernde farbige Lichtmuster.

Die vorliegende Arbeit geht nicht primär von Kommunikation aus, sondern von der notwendigen "Beziehungsarbeit" zur Aufrechterhaltung einer Fernbeziehung. Es geht also nicht darum, Kommunikation durch das Anreichern von Medien "emotionaler" zu gestalten, sondern um das Schaffen technischer Konfigurationen, die es dem Paar ermöglichen, aktiv über die Ferne an ihrer Beziehung zu arbeiten - auf freud- und bedeutungsvolle Weise. Ziel ist es, das psychologische Wohlbefinden in einer Beziehung $\mathrm{zu}$ erhöhen (siehe auch Bao \& Lyubomirsky 2013). Hintergrund herfür ist ein wohlbefindens- und erlebnisorientiertes Interaktionsdesign ("Experience Design", Hassenzahl et al. 2013), das sich zunächst mit dem psychologischen Wohlbefinden und dem Identifizieren bedeutungsvoller Erlebnisse und Alltagspraktiken beschäftigt, bevor es sich die Frage stellt, welche Technik in welcher Konfiguration sinnvollerweise zum Einsatz kommen könnte, um diese Erlebnisse zu ermöglichen.

Im Folgenden gehen wir zunächst kurz auf Fernbeziehungen ein und entwickeln aus dem bestehenden, überwiegend psychologischen Wissen, drei neue Ansatzpunkte für technikvermittelte Beziehungspflege über die Distanz: Fürsorge ermöglichen, Gemeinsamkeiten schaffen und Treffen planen. Zu jedem dieser Ansätze stellen wir dann ein eigenes Konzept vor. Jedes Konzept wurde als Prototyp realisiert und längsschnittlich vom Erstautor (Wei-Chi) und seiner Partnerin (Claire) im Sinne eines autobiographischen Gestaltungsprozess (Neustaedter \&Sengers 2012), gestaltet, erprobt und kritisch diskutiert. Wei-Chi und Claire leben seit vier Jahren in einer Fernbeziehung mit ca. $8550 \mathrm{~km}$ Distanz und einem Zeitunterschied von sechs bis sieben Stunden.

Der autobiographische Ansatz bedeutet, dass Gestalter im Gestaltungsprozess zunächst selbst Nutzer ihrer eigenen Konzepte sind und sie so über die Zeit kritisch entwickeln. Diesen Ansatz haben wir gewählt, weil so schon von der ersten Minute an im Kontext gestaltet wird. Auch grobe Konzeptideen können schnell skizzenhaft umgesetzt und ihr Einfluss auf das Beziehungsleben direkt erprobt werden, bevor sie überhaupt in einem Stadium sind, das es erlau- 
ben würde, externe Teilnehmern einzubinden. Gerade die Erprobung noch unausgereifter Prototypen über einen längeren Zeitraum ist hier wichtig, da aktuelle Ansätze des erlebnisorientierten Gestaltens oder des "persuasiven" Designs eigentlich immer davon ausgehen, dass sich Wirkungen erst über einen längere Zeitraum in der Lebenswelt der Nutzer einstellen. Das permanente Erleben des eigenen Entwurfs im Alltag fördert außerdem die Weiterentwicklung gewählter Gestaltungsansätze in bedürfnisorientierter Richtung (und nicht nur in technischer). So wird das Gestalten zum kontinuierlichen, lebensweltlich-informierten Verbesserungsprozess und zwar vom ersten Tag an. Natürlich können die Ergebnisse dieses Vorgehens nicht uneingeschränkt verallgemeinert werden. Führt man sich aber vor Augen, dass sein Kern die frühzeitige, längerfristige, genuine Nutzung von neuen, technischen Konfiguration in der Lebenswelt realer Menschen darstellt, wird doch schnell klar, dass dies kaum ein anderer Ansatz für sich reklamieren kann. Zumindest im Sinne der guten Praxis eines reflektierten Gestaltens scheint das Leben mit und das Aushalten des eigenen Entwurfs ein wichtiger erster Schritt.

Das Ziel des Beitrags ist zweigeteilt. Zum einen geht es darum, neue Ansatzpunkte für zukünftige Formen der technikvermittelten "Nähe auf Distanz" auf der Basis psychologischer Forschung zu romantischen Fernbeziehungen, also eher theoriegeleitet, zu identifizieren. Zum anderen haben wir damit begonnen, Konzepte zu entwickeln, umzusetzen und lebensweltlich zu erproben, die sowohl als Beispiele, als auch als Test dieser Ansatzpunkte dienen.

\section{Romantische Fernbeziehungen und neue Ansätze für das Gestalten unterstützender Technik}

Paare in Fernbeziehungen leben an zwei entfernten Wohnsitzen in eigenen Haushalten. Häufig ergibt sich eine solche Situation aus einer Berufstätigkeit oder dem Studium. Auch durch die deutlich effizientere Mobilität sind die Entfernungen in Fernbeziehungen größer geworden. Je nach Entfernung kann sich das Paar in kürzeren zeitlichen Abständen (z.B. einmal im Monat) oder selten (z.B. einmal im Jahr) treffen. "Echte" Präsenz ist beschränkt und ein für Fernbeziehungen typisches Lebens- und Kommunikationsmuster entsteht. Eine Fernbeziehung wird meist als Übergang verstanden. Paare in Fernbeziehung streben danach, später schließlich zusammen zu leben. Paare in Fernbeziehungen machen besondere Erfahrungen, wie z.B. intensive und lange Stunden der Konversation am Telefon, ein als besonders wertvoll empfundenes Zusammensein, emotionale Schwankungen beim Treffen und Abschied, sowie das Vermissen während des Alleinseins (Guldner 2006; Wendl 2013).

Zufriedenheit ist - wie in allen Beziehungen - auch bei romantischen Fernbeziehungen der Schlüssel zum Wohlbefinden und entsteht durch Beziehungspflege ("relationship maintenance behavior"). Wissenschaftler schlagen unterschiedliche Strategien im alltäglichen Miteinander vor, z.B. Positivität, Offenheit, Versicherung der gegenseitigen Wertschätzung, soziale Vernetzung und Aufgabenteilung (Canary 1991). Dies wird ergänzt durch Bewältigungsstrategien für entstehende negative Emotionen (Holt \& Stone 1988), besonders erzeugt durch wiederholt erlebte Einsamkeit. Aus einer langfristigen Perspektive ist eine gemeinsame $\mathrm{Zu}-$ 
kunft - Pläne, ein gemeinsamer Lebensentwurf - ein wichtiges Element, um die Beziehung aufrechtzuerhalten (Sahlstein 2006). Im Alltagsleben verbindet zwei auf Distanz Verliebte das Gefühl der Zusammengehörigkeit in ihrem jedoch selbstständigen Leben. Diese Verbundenheit muss durch Beziehungsarbeit allerdings immer wieder bestätigt werden (Le et al. 2010). Gemeinsame Pläne, das Teilen des Alltags, und die häufige Bestätigung der gegenseitigen Zuneigung über die Distanz sind also die zentralen Herausforderungen einer romantischen Fernbeziehung.

Zwei Verliebte in einer Fernbeziehung leben zwangsläufig in zwei unabhängigen, u.U. auch zeitlich asynchronen Lebenswelten. Das erschwert den alltäglichen, ritualisierten beiläufigen Austausch von Gefühlen und Gedanken (Stafford 2011; Wendl 2013). Ein gegenseitiges Füreinander-Dasein ist aber eine wichtige Voraussetzung für eine erfolgreiche Beziehung (Prager 1995, S. 257). Harvey und Omarzu (1999) konnten belegen, dass die Zufriedenheit von Paaren höher ist, je mehr die Partner bereit sind, sich um einander zu kümmern. Branham und Harrison (2013) bemängeln bei existierenden technikvermittelten Konzepten gerade die fehlenden Möglichkeiten der bewussten, reflektierten, fürsorglichen Interaktion. Die meisten Konzepte setzen auf das Gegenteil: beiläufige Gewahrwerdung. Konzepte für den Austausch von Nachrichten verstehen sich vorrangig als Medium und beschränken das Füreinander-Dasein auf die verbale Ebene. Im Alltag etwas für den Anderen zu tun, ist aber gerade eine Herausforderung für Fernbeziehungen. Aus diesen Überlegungen leitet sich der erste Ansatz für zukünftige Technik zur Unterstützung von Fernbeziehungen ab: das Ermöglichen von Fürsorge über die Distanz.

In einer Fernbeziehung spielen Gemeinsamkeiten eine wichtige Rolle. Sie sind zentral für stimulierenden Austausch (Canary 1991) und erzeugen Interdependenz (Le et al. 2010), auf die eine enge Beziehung gründet. Eine Fernbeziehung erfordert darüber hinaus allerdings gleichzeitig, selbständig zu sein und den eigenen Alltag zu organisieren (Wendl 2013). Dies unterstützt das Selbstwertgefühl und schützt auch vor Einsamkeit (Guldner 2006). Gemeinsamkeit und Selbstständigkeit führen unweigerlich zu Widersprüchen, wenn keine entsprechenden Praktiken aufgebaut werden. Ein zweiter Ansatz ist also, eine Gemeinsamkeit zu schaffen, die Interdependenz ermöglich, ohne die eigene Selbstständigkeit allzu stark einzuschränken. Das gemeinsame Kümmern um ein Haustier, eine Pflanze oder ähnliches ist eine typische Praktik, die eine solche, auch emotional fordernde Gemeinsamkeit erzeugt, ohne dass essentielle Aspekte der eigenen Selbstständigkeit aufgegeben werden müssen.

Fürsorgliches Handeln und eine Gemeinsamkeit überbrücken das Getrenntsein. Allerdings gibt es in jeder Fernbeziehung natürlich auch Phasen des physischen Zusammenseins, der "echten" Präsenz. Obwohl diese Phasen ein wahres Glückserlebnis für das Paar sein sollten, zeigen Studien, dass Paare sich besonders gestresst fühlen, wenn sie ihre gemeinsame Zeit nicht "besonders" intensiv oder "sinnvoll" verbringen (Westefeld \& Liddell 1982). Hier hilft ein wenig Planung (Gulden 2006), was allerdings nur selten vorher realisiert wird. Dabei könnte die Zeit der Trennung auch genutzt werden, um Bedürfnisse und Ansprüche an das nächste Zusammensein zu formulieren und notwendige Mittel vorher zu organisieren. Dies erzeugt nicht nur zukunftsorientierte Kommunikation (Pläne), sondern sowohl gemeinsame Aktivitäten/Aufgabeteilung, als auch Vorfreude und Stressreduktion. Gerade der empfangende Partner kann so seine oft ungute "Gastgeberrolle", die deutliche Verantwortlichkeiten mit 
sich bringt ("Hast Du eingekauft?", "Was kochen wir?", "Wo gehen wir heute Abend hin?"), ablegen. Als dritten Ansatz identifizieren wir also das Planen gemeinsamer Treffen.

Auf der Basis psychologischer Erkenntnisse über Fernbeziehungen haben wir drei Ansätze zur Gestaltung zukünftiger Technik zur Unterstützung von Fernbeziehungen identifiziert. Der erste Ansatz sucht nach Wegen, über die Distanz fürsorgliches Handeln zu ermöglichen. Der zweite schafft eine Gemeinsamkeit, trotz fehlender physischer Kopräsenz. Der dritte bereitet eine Phase des realen Zusammenseins vor.

\section{Beispielhafte Konzepte und ihre Erprobung}

$\mathrm{Zu}$ jedem Ansatz stellen wir nun ein beispielhaftes Konzept, sowie seine Erprobungen vor. Alle Konzepte sind funktionstüchtig. Allerdings sehen wir von einer detaillierte Darstellung der technischen Umsetzung ab, da diese für den vorliegenden Beitrag keine entscheidende Rolle spielt. In der kurzen Darstellung der Erprobung konzentrieren wir uns hauptsächlich auf Claire, da ihre Erlebnisse weniger beeinflusst durch Wissen über das Konzept scheinen, als die des Erstautors Wei-Chi.

Fürsorgliches Handeln im Alltag ist es beispielsweise, für den Anderen zu kochen oder aufzuräumen. Beides erscheint gleichzeitig als kaum mit vertretbarem Aufwand über die Distanz umsetzbar. Glücklicherweise wirken im Alltagsleben aber auch schon kleine, intime, eher symbolische Handlungen. Ein Beispiel ist es, das Licht für den Partner anzulassen, wenn dieser spät nach Hause kommt. Solche Gesten sind weniger komplex und durchaus mit Hilfe gängiger Technik auch über die Distanz zu ermöglichen. Wir haben dies zum Ausgangspunkt des Konzeptes Been There gemacht.

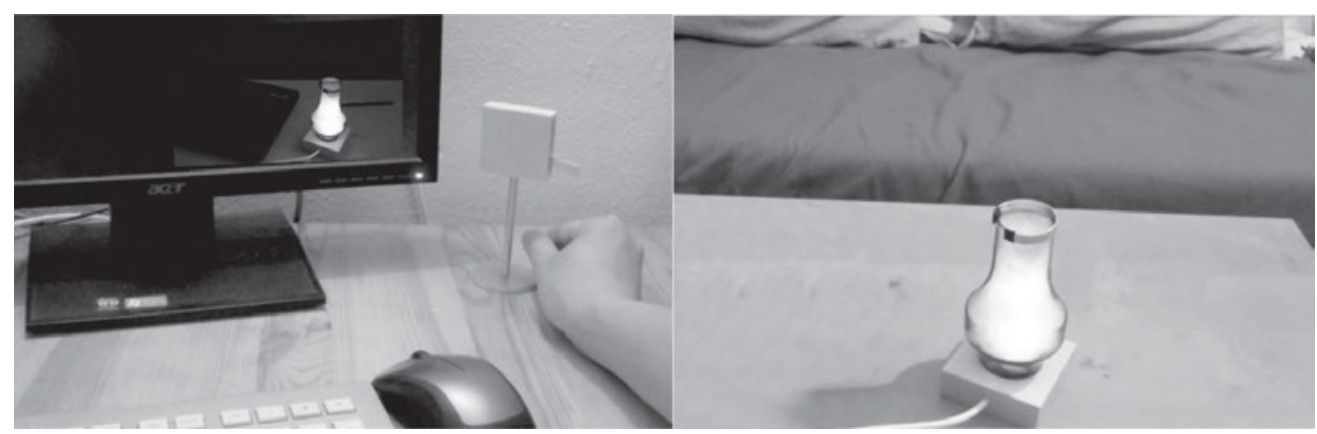

Abbildung 2: Been There

Been There besteht aus einer Nachttischlampe, die über das Internet geschaltet werden kann, und einer Kamera. Man kann die Wohnung des (abwesenden) Partners "besuchen" und für ihn dessen Nachttischlampe anschalten. Dies ist dann im Kamerabild zu sehen (Abbildung 2). Wenn der Partner später nach Hause kommt, weist das Licht darauf hin, dass der andere Sehnsucht hatte, "da" war, leider nicht bleiben konnte, aber als Geste der Fürsorge das Licht angemacht hat und so die Wohnung für den Anderen "vorbereitet". Dies Konzept ist sym- 
metrisch umgesetzt. Kamera und Lampe können ausgeschaltet werden, um die Privatsphäre zu schützen.

Das Konzept wurde in der in Abbildung 2 gezeigten Form von Claire und Wei-Chi für 84 Tage in ihrem Alltag genutzt. Für Wei-Chi wurde es zur Routine, Claires Wohnung nachmittags zu "besuchen", um die Zeit herum, wenn sie von der Arbeit nach Hause kommt. Claire besuchte Wei-Chi morgens, kurz bevor er aufstehen muss. Der "Besuch" und das Handeln in der anderen Wohnung erzeugte ein deutliches Gefühl von aktivem, fürsorglichem Handeln: "Dass ich auf dem Bildschirm sehe, wie ich die Lampe anschalte, erzeugt das Gefühl, dass ich es für den anderen mache" (Claire, 6. Tag). Gleichzeitig wird das angeschaltete Licht auch als fürsorglich erlebt: "Das Licht erzeugt ein warmes Gefühl, wenn ich spät nach Hause komme" (Claire, 29. Tag). Zusätzlich stellt sich eine symbolisch-informative Wirkung ein: "Ich komme nach Hause, sehe er hat mich vermisst, und ich spreche dann mit ihm via Skype" (Claire, 34. Tag) oder "ich weiß, dass sie heute Nachtschicht hat, weil sie mich vormittags [als ich noch geschlafen habe] besucht hat" (Wei-Chi, 16. Tag). Insgesamt wurde das Anschalten eines Lichtes als fürsorgliche, positiv und bedeutungsvoll erlebt. Been There wurde gut in den Alltag integriert und verlor auch über die Zeit nicht an Bedeutung. Allerdings spielte der "Besuch", also das Gefühl, sich in der Wohnung des Anderen aufzuhalten (d.h. die Kamera), eine wichtige Rolle. Konzepte sollten also zum einen eine Handlung ermöglichen (z.B. das Schalten einer Nachtischlampe), zum anderen aber auch eine Möglichkeit schaffen, relevante Zustände am andere Ort (also z.B. die Helligkeit) erlebbar zu machen. Probleme bezüglich der Privatsphäre sind selten aufgetreten. Ein Beispiel war: "Als ich sie [Claire] abends besuchte, war sie noch nicht ins Bett gegangen. Es war sehr spät in der Nacht und es war ihr unangenehm" (Wei-Chi, 20. Tag).

Als Beispiel der Förderung von Gemeinsamkeit über die Distanz haben wir uns am Bild des gemeinsamen Haustiers orientiert und das Konzept Furfur entwickelt. Furfur wohnt in einer Kiste, wobei eine "Verbindung" zwischen den Kisten es Furfur erlaubt, zwischen den Wohnorten der Partner ohne Zeitverzögerung zu wechseln (Abbildung 3). (Natürlich existiert je ein Furfur pro Kiste, aber immer nur eines darf aus der Kiste herausfahren.) Furfur reagiert auf Gesten der Zuneigung, auf Bewegungen und Klänge, indem es tanzt und die Stimme des jeweiligen Partners imitiert. Es speichert die erlernten Imitationen und reproduziert sie auch beim anderen Partner. Je mehr man mit Furfur interagiert, desto größer wird sein Repertoire. Das Verhalten Furfurs ist ein oft überraschendes Produkt der jeweiligen Interaktion beider Partner mit Furfur. Ähnlich wie bei einem gemeinsamen Haustier oder gar einem gemeinsamen Kind spiegeln sich die Eigenarten des anderen Partners wider und vermischen sich auf interessante Weise mit den eigenen Manierismen. So schafft Fufur eine Gemeinsamkeit, die Aspekte des Sich-Kümmerns, der emotionale Kommunikation, der Überraschung, aber auch der Sehnsucht miteinander verbindet. 


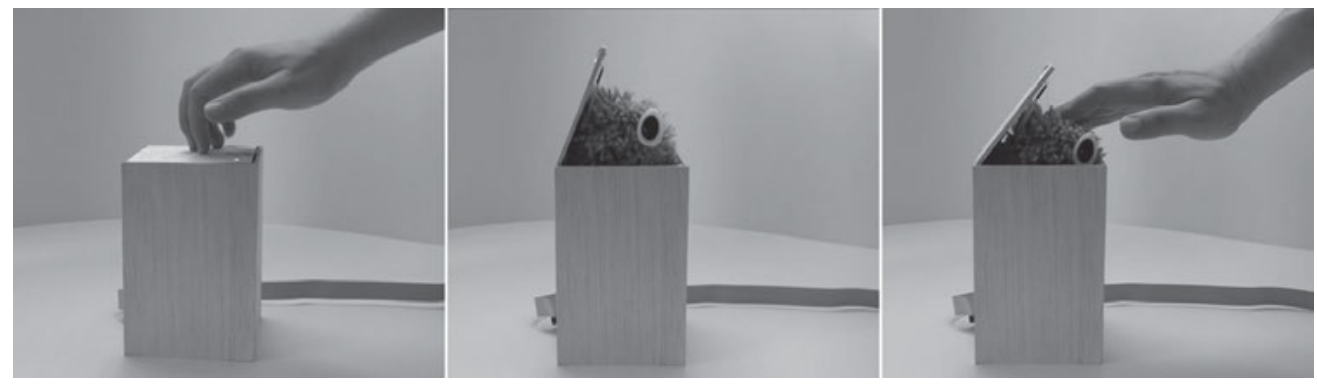

Abbildung 3: Auf die Kiste klopfen, um Furfur herbeizurufen (links); Furfur zeigt, was es sich abgeschaut hat (mitte); Furfur interagiert (rechts)

Die Konzeptidee von Furfur existiert in mehreren Versionen. Die erste Version wurde 84 Tage eingesetzt. Ein Gefühl der Verbundenheit entstand besonders dann, wenn Furfur beim Anderen war: "Ich sah, dass Furfur morgens nicht da war. Sie [Claire] hat ihn jetzt. Ich freue mich sehr, dass sie Furfur auch mag" (Wei-Chi, 10. Tag). Allerdings konnte Furfur in dieser Version noch keine Töne erzeugen. Das Imitieren des Anderen geschah durch Bewegung, was aber "nicht sehr deutlich" war (Claire, 12. Tag). Daraufhin entstand eine zweite Version, die bis heute verwendet wird (seit 67 Tagen), aber im Laufe der Zeit verbessert wurde. Furfur wird jetzt selbst aktiver und imitiert frech die Stimmen seiner Benutzer. "Es ist ein wunderschönes Erlebnis, Furfur zusammen mit Wei-Chi zu erziehen! Es ist wie ein elektronisches Haustier. Ähnlich wie mein Hund" (Claire, 28. Tag). Über Skype zeigen sich die Partner, wie sich Furfur beim Anderen benimmt, was ein Gefühl von Gemeinsamkeit erzeugt: "Ich freue mich in Skype zu sehen und zu hören, was Furfur dort macht" (Wei-Chi, 1. Tag) und Claire sagt: "Furfur verhält sich dort gut [bei Wei-Chi], mein Hund hier gut. Heute ist alles reibungslos" (Claire 13. Tag). Furfur hilft auch bei der Bewältigung von Einsamkeit: "Ich passe mich an die Anwesenheit von Furfur an. Ich habe einen neuen Mitbewohner" (Claire, 2. Tag). Manchmal bewirkt Furfur aber auch gerade das Gegenteil: "Furfur ist nicht hier. Wei-Chi auch nicht. Mein Hund auch nicht. Ich habe nur Edward Tulane in meinem Buch" (Claire, 32. Tag). Obwohl dies ein eher trauriger Moment zu sein scheint, zeigt er doch die emotionale Verbindung zwischen Furfur, Wei-Chi und Claire. Die Erprobung von Furfur zeigt, wie komplex entstehende Praktiken selbst bei einem künstlichen Haustier sein können. Furfur spielt nicht nur am eigenen Ort eine Rolle, sondern wirkt ganz besonders, wenn er gerade nicht da ist. Allerdings kann diese Wirkung emotional ambivalent sein: sie wirkt zwar immer verbindend, kann aber die Sehnsucht nach dem Anderen und damit auch Einsamkeitsgefühle steigern. Wichtig ist, dass das elektronische Haustier sowohl expressiv ist, also tatsächlich deutlich Manierismen und Eigenarten annehmen kann, als auch frech ist, also Gründe bietet, über es zu kommunizieren, um es gemeinsam "zu erziehen".

Um Treffen zu planen, haben wir als erstes Konzept eine Dating Box entwickelt. Jeder Partner schickt Ideen für gemeinsame Aktivitäten an die Box (das Gerät). Wenn das Pärchen sich später trifft, kann die Box geöffnet werden, um so aus den Anregungen zu schöpfen. Man kann allerdings zu jeder Zeit, also auch vorher, die Box öffnen und Ideen lesen, um z.B. einen Plan zu machen. Man kann auch Fragen, wie "Wo wollen wir einen schönen Kaffee trinken?", in die Box schicken, um den anderen zur Ideensuche zu motivieren, wobei eine der Fragen nach 
dem Zufallsprinzip auf dem Deckel angezeigt wird. So regt die Box dazu an, sich schon während der Trennung mit dem zukünftigen Zusammensein freudvoll auseinanderzusetzen, Möglichkeiten zu sammeln, Pläne zu schmieden und nimmt den Druck, sich zu einem bestimmten Moment etwas einfallen lassen zu müssen.

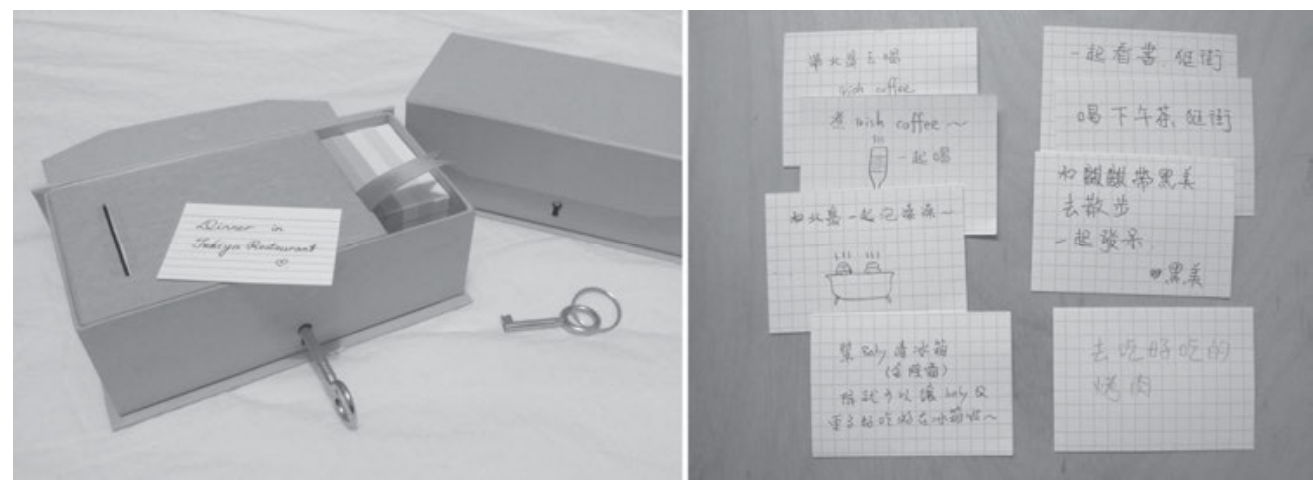

Abbildung 4: Die erste, analoge Version der Dating-Box (links) mit den gesammelten Ideen auf den Karten (rechts)

Die Dating-Box wurde in einer ersten analogen Form (also mit eingeschränkter "Funktionalität") von Wei-Chi und Claire zur Vorbereitung eines Treffens verwendet. Es gab einen Vorlauf von 90 Tagen. Das eigentliche Treffen dauerte 44 Tage. Insgesamt wurden im Vorfeld acht Karten (vier von jeder Person) erstellt, jeweils mit mehreren möglichen Aktivitäten. Acht Aktivitäten waren potentiell realisierbar und sieben wurden dann auch wirklich während des Treffens umgesetzt. Beispielsweise wurde die Idee, "gemeinsam baden zu gehen" durch eine Reise zu einer Thermalquelle umgesetzt. Es wurden auch unliebsame Alltagsaufgaben als Aktivität genannt, wie beispielsweise Claires Gefrierfach zu enteisen. Es war dann "ein besonders positives Erlebnis, als wir diese Aufgabe erfüllt hatten" (Wei-Chi). Trotz der acht Aktivitäten hatten Wei-Chi und Claire aber immer noch das Gefühl, nicht genug Ideen für stimulierende Aktivitäten zu haben. Drei Karten wurden geschrieben als die Box initiiert wurde, die anderen Karten wurden in der Woche vor dem Treffen in die Kiste eingeworfen. Zukünftige Versionen der Box werden versuchen, die Anzahl der Vorschläge zu erhöhen und eine kontinuierlichere Auseinandersetzung mit möglichen Aktivitäten zu fördern.

\section{Schluss}

Wir haben drei neue Ansätze für die Gestaltung von Technik zur Unterstützung romantischer Fernbeziehungen aus entsprechender psychologischer Literatur abgeleitet und beschrieben: Fürsorge ermöglichen, Gemeinsamkeiten schaffen, Treffen planen. Anders als viele andere (siehe Hassenzahl et al., 2012 für einen Überblick) setzen wir dabei bei der aktiven Beziehungsarbeit des Paares an und vermeiden es so, nicht nur bei der bloßen emotionalen Anreicherung von Kommunikation oder der Gewahrwerdung des Anderen zu verbleiben. Diese drei Ansätze sind natürlich weder erschöpfend, noch ausschließlich gemeint, sondern sollen zunächst als Anregung für andere Gestalter/Forscher dienen, das Thema der technischen Unter- 
stützung von Fernbeziehung auch aus diesen Perspektiven zu betrachten. Unsere eigenen Konzeptexplorationen, wie Furfur oder Been There, sollten als Beispiele dafür verstanden werden.

Die autobiographische Exploration der Konzepte zeigt Möglichkeiten und Schwierigkeiten. Fürsorge erfordert es, vor Ort, also im Lebensalltag des Anderen, etwas für diesen zu tun. Dies ist keine triviale Anforderung über die Distanz. Im Beispiel der Been There Lampe wird eine symbolische Handlung (das Licht für den anderen einzuschalten) zusammen mit einem "nach dem Rechten schauen" angeboten. Diese neue Möglichkeit wurde von Claire und WeiChi gut und nachhaltig in den Alltag integriert. Wichtig scheint gerade die Kombination von einem Blick in die Wohnung des Anderen und der Möglichkeit etwas zu tun. Probleme der Privatsphäre spielen bei einem romantischen Paar nur eine untergeordnete Rolle. Allerdings ist der Aktionsspielraum auf die Lampe beschränkt. Zukünftige Arbeiten sollten einen allgemeineren Ansatz finden, der es einem Paar erlaubt, aus einer breiteren Anzahl möglicher Aktionen zu wählen. Furfur als Beispiel für Gemeinsamkeiten wurde ebenso schnell als gemeinsam zu erziehendes "Haustierchen" angenommen. Zunächst war der stärkste Moment der Verbundenheit, wenn man wahrnimmt, dass Furfur gerade beim Partner ist. Erst nach einigem Ausprobieren konnte eine Interaktion erarbeitet werden, die es ermöglicht, dass sich beide Partner in Furfur wiedererkennen. Eine zukünftige Herausforderung ist es, das Verhalten von Furfur so weiterzuentwickeln, dass es noch stärker als Produkt gemeinsamer Beeinflussung erlebt wird. Treffen planen scheint ein wichtiges Themenfeld, allerdings waren die ersten Versuche mit der Dating Box eher enttäuschend. Es entstanden weder genug Vorschläge, noch regte sie in ihrer momentanen Form dazu an, sich zwischen den Treffen schon frühzeitig über mögliche Aktivitäten auszutauschen. Hier werden in Zukunft motivierende Elemente eine Rolle spielen, ganz besonders solche, die die Kommunikation über Aktivitäten im Vorfeld fördern.

Verfügbare Technologien erlauben es mittlerweile, schnell und verhältnismäßig einfach, funktionale Prototypen herzustellen. Umso wichtiger ist es, sich zukünftig auf die Qualität der zugrundeliegenden Konzepte zu konzentrieren. "Nähe auf Distanz" ist ein interessantes und vielversprechendes Anwendungsgebiet für Technik, und romantische Fernbeziehungen sind lediglich ein Teil davon. Allerdings sind gerade Fernbeziehungen sensible Konstrukte. Fragen, wo man konzeptionell ansetzt, wie Konzepte im Detail ausgestaltet werden müssen, wie sie sich dann im Alltag "benehmen" und integrieren lassen, und ob sie überhaupt Nähe und Wohlbefinden steigern, sind dabei zentral. Hiermit möchten wir einen Beitrag zu ihrer Beantwortung leisten.

\section{Danksagung}

Diese Arbeit wurde durch das Bundesministerium für Bildung und Forschung unterstützt (Projekt "Nähe auf Distanz", Förderkennzeichen 16SV7093K).

\section{Literaturverzeichnis}

Bao, K. J., \& Lyubomirsky, S. (2013). Making it last: Combating hedonic adaptation in romantic relationships. The Journal of Positive Psychology, 8, 196-206. 
Branham, S., \& Harrison, S. (2013). Designing for collocated couples. In C. Neustaedter, S. Harrison, $\&$ A. Sellen (Eds.), Connecting Families. Springer.

Canary, D. J., Hause, K. S., Stafford, L., \& Wallace, L. A. (1991). An inductive analysis of relational maintenance strategies. Communication Research Reports, 10, 5-14.

Gallace, A., \& Spence, C. (2010). The science of interpersonal touch: an overview. Neuroscience and Biobehavioral Reviews, 34, 246-59.

Guldner, G. (2006). Long distance relationships: The complete guide. 4. Auflage. JF Milne.

Harvey, A. J. H., \& Omarzu, J. (1999). Minding the close relationship: A theory of relationships enhancement. Cambridge University Press.

Hassenzahl, M., Heidecker, S., Eckoldt, K., Diefenbach, S., \& Hillmann, U. (2012). All you need is love: Current strategies of mediating intimate relationships through technology. ACM Transactions on Computer-Human Interaction, 19, 1-19.

Hassenzahl, M., Eckoldt, K., Diefenbach, S., Laschke, M., Lenz, E., \& Kim, J. (2013). Designing moments of meaning and pleasure. International Journal of Design, 7(3), 21-31.

Holt, P. A., \& Stone, G. L. (1988). Needs, coping strategies, and coping outcomes associated with long-distance relationships. Journal of College Student Development, 29, 136-141.

Kowalski, R., Loehmann, S., \& Hausen, D. (2013). cubble : A multi-device hybrid approach supporting communication in long-distance relationships. In Proceedings of TEI '13, 10-13.

Le, B., Korn, M. S., Crockett, E. E., \& Loving, T. J. (2010). Missing you maintains us. Journal of Social and Personal Relationships, 28, 653-667.

Lee, K. M. (2004). Presence, Explicated. Communication Theory, 14, 27-50.

Neustaedter, C., \& Sengers, P. (2012). Autobiographical design: what you can learn from designing for yourself. Interactions, 19, 28-33.

Prager, K. J. (1995). The psychology of intimacy. Guilford Press.

Sahlstein, E. M. (2006). Making plans: Praxis strategies for negotiating uncertainty-certainty in longdistance relationships. Western Journal of Communication, 70, 147-165.

Stafford, L. (2011). Maintaining long-distance and cross-residential relationships. Routledge.

Strong, R., \& Gaver, B. (1996). Feather, scent and shaker: supporting simple intimacy. In Proceedings of CSCW'96, 16-20.

Wendl, P. (2013). Gelingende Fern-Beziehung - entfernt zusammen wachsen, 6. Auflage. Herder.

Westefeld, J. S., \& Liddell, D. (1982). Coping with long-distance relationships. Journal of College Student Personnel, 23, 550-551.

\section{Kontaktinformationen}

Dipl.-Des. Wei-Chi Chien, Prof. Dr. Marc Hassenzahl, Dipl.-Des. Eva Lenz

Erlebnis und Interaktion, Fachbereich Gestaltung

Folkwang Universität der Künste, Essen.

www.marc-hassenzahl.de 